\title{
A Narrative Review on Haptic Devices: Relating the Physiology and Psychophysical Properties of the Hand to Devices for Rehabilitation in Central Nervous System Disorders.
}

\author{
SARA DEMAIN ${ }^{1}$, SARAH. E. CUNNINGHAM ${ }^{1}$, CHERYL D. METCALF $^{1 \& 2}$, DEYI ZHENG $^{2} \&$ \\ GEOFF. V. MERRETT ${ }^{2}$
}

${ }^{1}$ Faculty of Health Sciences, University of Southampton

${ }^{2}$ Electronics and Computer Sciences, University of Southampton

Implications for Rehabilitation

- The current lack of emphasis on somatosensory rehabilitation may contribute to poor hand recovery in patients with central nervous system pathology.

- Haptic technologies have the potential to improve sensation and sensory motor integration in neurological conditions

- An underlying theoretical rationale is provided for the design and clinical use of haptic technologies in neuro-rehabilitation of the hand.

\begin{abstract}
Purpose. This paper provides rehabilitation professionals and engineers with a theoretical and pragmatic rationale for the inclusion of haptic feedback in the rehabilitation of central nervous system disorders affecting the hand.

Method. A narrative review of haptic devices used in sensorimotor hand rehabilitation was undertaken. Presented papers were selected to outline and clarify the underlying somatosensory mechanisms underpinning these technologies and provide exemplars of the evidence to date.

Results. Haptic devices provide kinaesthetic and/or tactile stimulation. Kinaesthetic haptics are beginning to be incorporated in central nervous system rehabilitation, however, there has been limited development of tactile haptics. Clinical research in haptic rehabilitation of the hand is embryonic but initial findings indicate potential clinical benefit.

Conclusions. Haptic rehabilitation offers the potential to advance sensorimotor hand rehabilitation but both scientific and pragmatic developments are needed to ensure that its potential is realised.
\end{abstract}

Keywords: Haptic, Hand, Sensory, Motor, Rehabilitation, Virtual Reality.

\section{Introduction}

This paper provides rehabilitation professionals and engineers with a theoretical and pragmatic rationale for the inclusion of haptic feedback in neurorehabilitation of the hand.

The term haptics, derived from the Greek words haptikos and haptesthai, means pertaining to the sense of touch [1]. Haptic feedback is the provision of somatosensory stimuli via physical interfaces to provide tactile or kinaesthetic information to the device user. In recent years the field of haptics has contributed significantly to our understanding of i) how touch sensation is used to explore and understand the environment and ii) the role of sensation in functional movement. Haptic devices, capable of generating touch sensations and of creating virtual objects have been developed [2]. Everyday examples include the use of vibration devices in mobile phones and resistive "force feedback" in computer gaming joysticks. It is the potential of such haptic devices to facilitate 
hand function rehabilitation in people with central nervous disorders that is the focus of this paper.

A literature search for papers which referred to the use of haptic technologies, both kinaesthetic and tactile, in the rehabilitation of the upper limb and hand following central nervous system disorders such as stroke, multiple sclerosis, and spinal cord injury was conducted. This paper is not a systematic review of the evidence. Rather, it presents exemplar papers to provide a narrative overview of the state of the art in terms of i) current haptic technologies, ii) a rationale for their use in neurological rehabilitation of the hand and iii) future directions for haptic development.

Over the last 10 years, research on the use of haptic devices in neurorehabilitation has begun to emerge [3]. Most of this research has been conducted in people with stroke although some evidence in conditions such as multiple sclerosis and spinal cord injury is available [4]. Studies have incorporated haptics into computerised virtual reality (VR) systems. These papers present compelling arguments for the value of haptically enhanced VR suggesting it has the potential to enhance motor-task training by providing repetitive, goal orientated rehabilitation to promote long-term neuroplasticity and improved motor control [5]. They also claim that traditional one-to-one therapy is rarely able to provide the necessary intensity of training; a situation that is set to worsen given the anticipated increase in neuro-disability as the population ages. The inclusion of gaming in VR environments, it is argued, can also enhance user motivation by increasing attention, providing reward and offering feedback on success [6]. VR systems can also capture detailed kinetic and kinematic data which, it is reasoned, can be used to adapt task complexity and to enable therapists to monitor progress [7]. $\mathrm{VR}$ is, therefore, viewed as an important adjunct to therapy.

Whilst these arguments for the use of VR in neurorehabilitation are compelling, the specific justification for the inclusion of haptic rehabilitation is not clearly made by these authors. Moreover, there has been little attempt to link the psychophysical properties of the hand to the use and design of haptic devices for hand rehabilitation. We propose that an understanding of i) the somatosensory systems and ii) sensory-motor integration can provide rehabilitation professionals and engineers with a useful framework for the development and evaluation of devices for haptic-rehabilitation in neurology.

This paper therefore:

- Outlines the key somatosensory mechanisms, their importance to functional movement and the problems that arise as a result of central nervous system disorders which result in motor and somatosensory impairment of the hand

- Provides a rationale for the inclusion of haptic technologies in the rehabilitation of neurologically impaired hand function.

- Describes currently available haptic technologies for hand rehabilitation and provides an outline of research evidence into their benefits.

- Discusses ideas for future directions in research and device development in rehabilitation haptics.

\section{The somatosensory systems and sensory motor integration (motor control)}

People use their hands almost constantly to explore their environment and the objects in it. It is not surprising, therefore, that the hand has the largest sensory and motor representations in the sensory cortex [8]. People with normal sensation and movement can usually identify a familiar object by touch alone. This skill which, for instance, enables people to put a hand into a pocket and select the correct value coin from a variety of small flat discs using touch alone, is known as hapticgnosis or stereognosis [9].

Stereognosis is a highly complex sensori-motorperceptual skill. It entails active haptic sensing 
[8]; incorporating both the motor control to move the hand around in the pocket and manipulate the various discs with the fingers, and the sensory capacity to detect a range of physical properties such as the temperature, size, shape and smoothness. There are two main somatosensory systems; both are vital to stereognosis. The two systems are known as i) the kinaesthetic (also called proprioceptive) system which provides information about the position and movement of the body and limbs, and ii) the tactile (also called cutaneous) system which provides feedback from the external world. In the task above, the kinaesthetic system provides feedback on the position of the fingers and hand enabling the shape and size of the various discs to be perceived. The cutaneous system responds to the objects' thermoconductive properties (is the disc metal or plastic?), texture (is the disc smooth or embossed?) and the pressure it exerts during manipulation pressure (is the coin round or does it have corners?). Additionally, stereognosis entails complex cognitive processes which i) enable the integration of the sensory information about the object's physical properties; ii) compare it with stored information about the properties of coins, buttons and tokens and iii) recognise and name the held object.

Having demonstrated the complexity underpinning everyday sensory-motor tasks the somatosensory systems are discussed in more detail.

\section{Kinaesthetic somatosensory system}

Kinaesthetic or proprioceptive awareness is obtained from a variety of mechanoreceptors which provide information about the position and movement of joints and the length and tension of muscles. Quick adapting Pacinian corpuscles (FAII), found in joint capsules and ligaments are maximally active during movement, whilst slow adapting Ruffini corpsucles (SAII) in individual ligaments provide information on joint position and are maximally sensitive at the end of joint range [10]. Muscle spindles primarily provide information about muscle length and velocity
[11] whilst golgi tendon organs provide information on muscle tension [10]. Nocioceptive free nerve endings in muscles, ligaments and capsules are sensitive to touch, pressure and pain and are especially active in prolonged stretch [10]. Mechanoreceptors (SAII and FAII) in the skin also contribute to kinaesthetic sense when the skin over both small and large joints is stretched [12]. Kinaesthetic sense declines with age [13], joint diseases [14] and injury [15]. It is also impaired by damage to the central and peripheral nervous systems. Kinaesthetic sense is trainable and is enhanced in athletes [16], dancers [17] and musicians [18].

\section{Tactile somatosensory system}

The tactile or cutaneous somatosensory system conveys what is, in lay terms, called the sense of touch. It transmits information about various physical parameters, mostly those that relate to the surface properties of objects [8], thus enabling people to determine whether items are hot, cold, sharp, blunt, soft, rigid, etc. The tactile system also responds to physical parameters which are capable of generating tissue damage by generating a perception of pain. The receptors of the tactile system are found in the skin over the entire body but are most prevalent in the hand, lips and genitals [8].

Tactile afferent fibre endings in skin can be divided into mechanoreceptors, thermal receptors and nocioceptors. There are four main classes of mechanoreceptors in the glabrous skin of the hand. These are the fast adapting (FA) Meissner corpuscles (FA-I) and Pacinian corpuscles (FA-II) and the slow adapting (SA) Merkel Cell Neurite Complexes (SA-I) and Ruffini Endings (SAII) [8] [19]. Stimulation of FA receptors is associated with the perception of vibration, whereas activation of SA units is associated with perceptions of pressure, position and skin displacement [20]. Overlapping activation thresholds mean that a given stimulus generally activates more than one class of receptor [21]. Thermoreceptive units are divided into two groups, warm and 
cold receptors, the latter of which are more numerous throughout epidermal and dermal skin layers [8]. Cold receptors respond to temperature decrements, showing peak sensitivity at skin temperatures of $25^{\circ} \mathrm{C}$, although they also respond to temperature ranges of $5-43^{\circ} \mathrm{C}$. Warm receptors are responsible for detecting temperature increases and are maximally sensitive at $45^{\circ} \mathrm{C}$ [22]. Nocioceptors signal the potential for tissue damage. Mechanical nociceptors respond to excess pressure, mechanical deformation or skin puncture; thermal nociceptors fire when temperatures exceed $45^{\circ} \mathrm{C}$ or fall below $13^{\circ} \mathrm{C}$ [23] whilst chemical respond to certain environmental irritants.

Tactile somatosensory information is vital for normal grasping activities, for instance, tactile information about surface texture and object shape [24] is vital to ensuring correct grasp pressures. Tactile information is also necessary for tasks requiring rapid dextrous finger movement such as piano [25] or clarinet playing [26]. The absence of normal tactile feedback results in clumsiness in daily activities [27].

\section{Somatosensory impairments in disorders of the central nervous system and the impact on movement and function.}

Somatosensory impairment is common in many neurological disorders and contributes to the functional deficits observed in these conditions. Losses can occur in each or all of the kinaesthetic or tactile domains. Reports suggest that up to $85 \%$ of people with stroke have somatosensory deficits in the 'affected' limbs [28] and between 12-26\% of people also have deficits in the 'unaffected' limbs [29].

Kinaesthetic and tactile sensation is also frequently lost or impaired in people with spinal cord injuries, multiple sclerosis and cerebral palsy. Neuroanatomy and neurophysiology studies have demonstrated the key roles of both the kinaesthetic and tactile systems in normal human movement [30] [31] [32]. In addition, damage to somatosensory regions has been shown to generate movement abnormalities.
Damage to the somatosensory systems of the cerebellum and the dorsal columns of the spinal cord in Multiple Sclerosis results in ataxia [33]. Similarly, strokes in the thalamus [34], sensory cortex (in the parietal lobes) [34], or dorsal pontine area of the brain stem [35] cause both sensory loss and disordered motor control. Somatosensory loss has been associated with uncoordinated movement at the shoulder, elbow and hand [36], ineffectual regulation of grip forces [37], impaired motor learning [38] and reduced functional recovery [39]. Collectively, this evidence of the importance of somatosensory-motor integration to motor function provides an explicit theoretical rationale for the inclusion of haptic devices into neurorehabilitation.

The integral connection between somatosensory and motor function is clear. Recent advances demonstrate that the relatively poor recovery rates seen in the hand after stroke [40] [41], are associated more with sensorimotor integration deficits than with impairments of strength or tone. It is surprising therefore, that clinical neuro-rehabilitation has paid relatively little attention to understanding and treating the sensory components of neurological disease [42], nor to utilising sensory feedback in the restoration of motor function [28]. One of the key roles of the hand is as a sensory organ, yet people with poor motor function, who are unable to grip or grasp, receive little stimulation of the palmar surfaces. It is possible that this lack of afferent input may be a significant factor in the poor recovery rates currently seen in the hand. A recent review [43] and meta-analysis [44] of sensory stimulation after stroke identified a paucity of high quality studies and inconclusive evidence for the benefits. Despite this, individual studies indicate the potential of tactile stimulation in the restoration of sensation in stroke [28] [45], MS [46], incomplete spinal cord injury [47] and of motor control in stroke [45] and spinal cord injury [47]. High quality research into sensory feedback in neurological conditions is urgently needed. Haptic technologies offer the potential to provide somatosensory stimulation during motor tasks 
and for increasing the amount of afferent input to the hand.

\section{Haptically Enhanced Neurorehabilitation}

The focus in haptic research has been on devices which have been designed with the primary intention of generating somatosensory feedback. These, which we define as primary haptic devices, are the focus of this paper. There are, however, other devices and techniques used in neurological rehabilitation which do not have somatosensory stimulation as a primary aim but nevertheless provide sensory feedback by promoting motor activity. For instance, devices such as robots and traditional therapist facilitated movement may be aimed at generating movement but will also provide sensory feedback. By enabling people to move in a purposeful manner, and to achieve motor goals, such devices and techniques automatically generate secondary haptic stimuli. It is important to remind rehabilitation engineers and therapists that such devices not only generate functional gains via improvements in muscle strength or joint range but may also facilitate motor learning through enhanced sensory-motor integration. Having highlighted this important issue we do not plan to discuss such secondary haptic stimuli further, but rather to focus on the primary haptic devices.

The following sections therefore present how haptic devices developed for hand rehabilitation relate to the two key somatosensory systems (kinaesthetic and cutaneous) and describe evidence on the benefits of haptic hand rehabilitation.

\section{Kinaesthetic Haptic Rehabilitation}

The majority of haptic devices for hand rehabilitation focus on stimulating the kinaesthetic system. Kinaesthetic devices generate forces which guide, resist or perturb movement, providing force feedback about the physical properties and/or movements of virtual objects produced by a computer. Inclusion of kinaesthetic haptic feedback has been demonstrated to improve the speed and precision of performance over virtual reality alone [48].

There are various mechanisms for providing force feedback, including mechanical levers or pneumatic actuators connected to the hand, which form an interface between the person's fingers and a computer. Finger movements are translated into actions on the screen and feedback is provided to the fingers through the mechanical levers [49]. These forces activate the kinaesthetic afferents in the ligaments and capsule, the muscle spindles and golgi tendon organs. This generates a perception of the virtual physical properties of the object so that the user is able to "feel" the virtual object's size, weight or texture.

Force-feedback kinaesthetic information can be experienced using a robotic device, forcefeedback stylus or data glove with exoskeleton [50]. Hand and wrist exoskeletons, for example HWARD [51] and PERCRO [52] can be used to monitor, and impose movement at the individual joint level; however, they are complex and expensive and consequently not suitable for use in home therapy. Smaller exoskeleton systems include the Cyberglove [53], but these remain expensive for individual purchase. Endpoint devices, including the MITManus [54] and the 2DoF Haptic Knob [55], and the 6 DoF Phantom [56] are simpler and therefore less expensive to implement.

The Phantom permits simulation of fingertip contact with virtual objects. A pen like stylus tracks the pitch, roll, and yaw and $\mathrm{x}, \mathrm{y}$, and $\mathrm{z}$ Cartesian coordinates of the virtual point-probe. Its actuators communicate forces back to the user's fingertips as it detects collisions with virtual 3-D objects, simulating the sense of touch [57]. The Phantom is being used as a training device for simulating tasks such as surgery [58] which require tool use (e.g. scalpel) in the 'real' world. However, the validity of such end-user devices in neurorehabilitation needs careful consideration. They may be useful for re-educating tasks such as cutting or cleaning teeth which require tool use but have less transferability into other 
activities of daily living (e.g. dressing, eating an apple) where the hand comes into direct contact with the object of interest. This is an important issue for future research.

In recent years empirical evidence has begun to accumulate on the potential benefits of kinaesthetic haptic devices for neurorehabilitation of the hand.

Alamri et al [1] developed a haptic interface which utilised the CyberGrasp glove to perform VR activities based on functional tasks from the Jebsen Test of Hand Function and the Box and Block Test. They captured data from healthy people whilst interacting with their system and showed its potential to capture key performance metrics for quantitatively evaluating task performance. They proposed establishing a data base of reference material from healthy people against which the performance of neurological patients could be compared. If realised, this offers opportunities for increased precision in functional measurement and may lead to a better understanding of training and recovery mechanisms.

Adamovich et al [7] demonstrated improvements in finger range, speed and strength of movement in several participants trained using both the CyberGlove and Rutgers Master II-ND haptic glove in a VR environment. Their pilot study trained eight people, who were at least one year post-stroke and were able to actively extend their hemiplegic wrist at least $20^{\circ}$ and extend the metacarpophalangeal joints at least $10^{\circ}$. People practiced a range of VR generated exercises combined with force feedback for 2-2.5 hours per day, 5 days per week, for 3 weeks. Six people demonstrated significant improvements in finger and thumb range, four people in finger speed and 3 in finger strength. The training was also generalised to functional movements with all participants demonstrating functional improvement in terms of reduced completion times in the Jebsen Hand Test. These interesting results need to be interpreted cautiously, however, given the lack of control group. The variations in improvement observed in this heterogeneous sample also need to be better understood with larger and/or more homogenous samples.

Merians et al [59] investigated the use of computerised haptic enhanced VR training on the hemiparetic hand of eight participants poststroke. Each participant received 2-21/2 hours of training per day over a three-week period and were measured using the Jebsen hand function test, plus computerised measurement of finger and thumb range of motion, velocity and fractionation. The results showed an improvement in the both the Jebsen test and all computerised measurements, and those improvements were shown to be retained after a 1-week follow-up.

Qui et al [60] used VR haptic training regimes to try and understand the mechanisms of arm and hand recovery in stroke. They used the CyberForce and CyberGlove to provide force feedback to the hand and the Haptic MASTER (Moog NCS, The Netherlands), a force controlled robot for the arm. They developed VR simulations for the hand alone, the arm alone, and the hand and arm together. People with stroke were allocated to train either: the hand and arm simultaneously $(n=7)$; the arm alone; or, the hand alone $(n=11)$ for 3 hours per day for 8 days. The results indicated greater improvement when the hand and arm were trained together suggesting differing patterns of motor learning between the different training approaches. Despite the limitations of small sample size, lack of random allocation and short training period the potential for such devices to explore hand and arm recovery mechanisms requires further exploration with more robust designs.

Although evidence from healthy populations has demonstrated the benefits of haptic enhanced VR over VR alone [45] [61] [62], we were unable to identify any similar research in neurorehabilitation. This means that whilst haptic enhanced kinaesthetic rehabilitation appears promising, it is impossible to ascertain the additional contribution that the haptic elements make over and above those that would 
be gained from non-haptic VR. This is an important area for future research.

\section{Tactile haptic rehabilitation}

Several modalities have the potential for providing tactile haptic stimulation. Tactile devices may operate via a number of mechanisms including the application of an electric or magnetic field, by incorporating piezoelectric crystals, shape memory alloys or pneumatic systems, or the use of fluids which change viscosity in different environments. These devices primarily operate by applying pressure, vibration, electrical fields or changes in thermal flow. As a result, tactile displays have been described as being capable of closely replicating the tactile parameters of an object including temperature, texture, shape and roughness [63]. Used alongside computerdisplayed virtual reality environments, tactile haptic systems can provide experience of completing everyday interactions with the environment [49].

Most neurorehabilitation haptic devices have focussed on providing kinaesthetic feedback; few have utilised tactile stimulation and those that have, have focussed on vibrotactile or electrotactile stimulation. The ability to exploit sensory substitution phenomena by applying vibrotactile or electrotactile stimuli on sensate regions of skin (e.g. unaffected limb or torso) has been demonstrated to improve grip force regulation in people with spinal cord injury [65] and Mutiple Sclerosis [66], although these are small studies and the longer term functional benefits have yet to be demonstrated. The application of tactile haptic stimuli to rehabilitate insensate limbs has been less well explored. There is, substantial potential for incorporating tactile devices into neurorehabilitation of hand function, but it is essential that the psychophysics of tactile haptic perception are given full consideration in the design of future systems.

\section{Vibrotactile stimulation}

Cutaneous detection of vibration is dependent on the frequency of the delivered vibration [66].
Activation of the FA-I afferent receptors is associated with the perceptual sensation of a flutter, rather than the deep and diffuse feeling that tends to follow FA-II activation [67]. FA-II afferents are extremely sensitive to high frequency stimulation due to the structure of the afferent ending and the layers of connective tissue surrounding them. This ensures that only high frequency stimulation reaches the afferent nerve [67]. FA-II afferent units, responsible for sensing vibrotactile feedback, have a large receptive field that may encompass whole regions of the fingertip [8] and account for $40 \%$ of the glaborous skin mechanoreceptors [68]. These large receptive fields mean that FA-II units have virtually no spatial resolution and that vibrating stimuli are resolved across the entire receptive region. There is therefore no advantage in providing multiple points of stimulation to the fingertip; one actuator per fingertip is considered sufficient [69], with optimal sensitivity achieved at approximately $250 \mathrm{~Hz}[70]$.

The waveform of the applied stimulation also influences its perception. Square waves provide the most intense feeling of vibration, whereas sine waves are perceived to be the smoothest, with triangle waveforms somewhere in the middle [71]. Prolonged exposure to vibratory stimulation has been associated with long-term nerve and tissue damage through excessive use of games controllers [72] and machinery [73]. Haptic systems might consider incorporating a user-control system for determining stimulation intensity in addition to avoiding prolonged usage.

Vibrotactile stimulation is used widely throughout gaming, virtual reality, and telecommunications. Controllers for the Sony PlayStation and the Nintendo Wii incorporate vibrotactile feedback to enhance the gaming experience [74], [75]. The CyberTouch, developed by CyberGlove Systems [53], has been used in neurorehabilitation research to provide vibrotactile stimulation to the palm and the dorsum of the fingers, whilst the user interacts with a virtual environment [7]. It is difficult, however, to draw conclusions about 
the effectiveness of vibrotactile stimulation from these studies, as it was always used in conjunction with kinaesthetic force feedback. Moreover there is little logical rationale for stimulating the dorsum of the fingers when most tactile object interaction occurs via the volar surface. More recently vibrotactile feedback has been used to enhance user interaction in touch-screen interfaces in devices such as the iPad [76]. Despite these vibrotactile applications, tactile haptic devices are not routinely included as part of neurological rehabilitation.

Vibrotactile feedback is only one method of providing cutaneous stimulation and is not the only solution for targeting the FA-II afferent receptors. Advances in ultrasound interfaces are also able to provide gross feedback to an individual's hand when interacting with a virtual interface or computer screen [77].

\section{Pressure}

Pressure is perceived by the low-frequency receptive ability of the SA-I afferents. In clinical settings, sensitivity to pressure is often used as a measure of absolute tactile sensitivity. Pressure sensitivity is inferred by asking individuals to close their eyes and applying nylon monofilaments calibrated to deliver predetermined forces to ascertain the minimum perceivable force applied to the skin. Within the context of hand rehabilitation, absolute sensitivity thresholds for the fingertips and sole of the foot have been reported as $0.63 \mathrm{~g}$ and $3.5 \mathrm{~g}$ respectively [78]. Sensitivity to light touch is also used as a screening tool for detecting neuropathic damage. Individuals, who are unable to perceive a $10 \mathrm{~g}$ force, when applied to the skin, should be investigated for potential underlying neuropathological damage

Tactile haptic devices have been developed that capitalise on pressure sense. These include:

- Devices that use a motor to constrict a band worn around the fingertip[49] [79];

- Devices utilising electrorheological or magnetorheological fluids that change their viscosity [80];
- Ultrasound, which may also target the FA-II afferents generating light touch [81]; and

- Shape memory alloys that change shape when an electric current is passed through the alloy thus constricting the finger and generating pressure [49] [82] [83].

When developing haptic devices to target the SA-I afferent receptors activated through pressure, it is worthwhile considering characteristics of the user-population. Technologies used for neurorehabilitation may choose to ensure that devices are capable of delivering a range of forces, up to and exceeding $10 \mathrm{~g}$, to increase the likelihood that they are perceivable by individuals with somatosensory neuropathic damage. Increased depth of skin indentation may also be explored as a means of delivering cutaneous sensations.

The effectiveness of haptic pressure devices as a tool for rehabilitating sensation and improving motor function, in people with neurological disease, has yet to be demonstrated. Recent research [49] has however, tested the feasibility of using these devices by exploring the sensations healthy people $(n=7)$ and people with stroke $(n=4)$ perceived when wearing i) motor constrictor and ii) shape memory alloy devices This study illustrated that pressure applied simultaneously to the volar pads of the thumb and index fingers generated a perception of holding something, indicating the potential for these devices to be used to simulate objects in VR environments. This sensation was, however, only reported by one of the people with stroke; a man who had only minor loss of light and deep touch.

\section{Temperature}

The design of devices for providing thermal haptic stimulation needs to consider the thermal range to be applied in terms of which receptors are stimulated and whether the stimulation will be painful or cause thermal damage. They also need to consider that the ability to detect temperature change is influenced by the rate of change. Providing that a temperature remains 
within the neutral range of $30-36^{\circ} \mathrm{C}$, individuals may not notice changes of up to $5^{\circ} \mathrm{C}$ if the rate of change is less than $0.5^{\circ} \mathrm{C} / \mathrm{min}$ [84]. Thermal sensitivity is also dependent on a person's age, and the area of skin being tested. The skin also has poor spatial resolution with regards to detecting temperature change [8]. Information about an object's thermal properties may facilitate object identification when textual or visual information is unavailable, for example stereognosis [84]. However, it is possible that spatial summation of temperature change may mask the intended simulation of fine object properties in devices aiming to provide both types of sensory information. In addition to this, since age is known to impact on thermal sensitivity, it is not sufficient to rely on self report as a measure of detecting temperature increase. Any technologies must be known to operate within a temperature range considered comfortable by control participants before being used with clinical groups. Merrett et al [49] demonstrated that the temporal modulation provided by shape memory alloys created a perception of holding a warm object which may be useful for simulating objects such as a cup of tea.

\section{Perception of Electrocutaneous Stimulation}

Electrotactile stimulation involves passing an electrical current through the skin, targeting afferent fibres, to evoke tactile sensations, rather than activating muscle receptors or unmyelinated $\mathrm{C}$ fibres [85]. The location of applied stimulus affects the absolute threshold, i.e. the smallest electrical current that can be detected due to the varying thickness of skin layers across the body. Absolute threshold is also affected by the repetition rate, the waveform and the type and configuration of the electrode, including its material and the type of electrode paste. The electrical resistance of skin is affected by its moisture level, with resistance levels of $50-200 \mathrm{k} \Omega$ reported when dry, but less than $10 \mathrm{k} \Omega$ when hydrated [85]. The perceptual threshold for electrocutaneous detection on the thumb surface has been documented as $1.5 \mathrm{~mA}$ delivered at $3 \mathrm{~Hz}$, producing a two point discrimination distance of approximately $5.6 \pm$ $1 \mathrm{~mm}$ [86], targeting both the FA-I and FA-II afferent receptors. Cutaneous electrostimulation has demonstrated positive effects on motor performance, limb sensation and the configuration of Sensory Evoked Potentials of the paretic limb in people with chronic stroke [87]. However, a high degree of variability in the perception of electrocutaneous stimuli has been reported, with qualitative reports of electrotactile sensations ranging from tingles to burning pain, depending on the voltage and current applied; this variability has also been reported in individual participants between sessions [87]. One caveat in the use of this type of stimulation is that the distinction between detection of electrical stimulation and pain perception is, on average, only $15 \mathrm{~dB}$, which contrasts to a dynamic range of $60-80 \mathrm{~dB}$ for vibrotactile stimulation [88]. The likelihood that an individual will experience pain in response to electrocutaneous stimulation increases if the skin is dry. Varying the position of the electrodes as little as $1 \mathrm{~mm}$, can also affect sensation perception, and so a consistent approach is essential. This variability in perceived sensation has hindered the development of electrotactile actuators for stroke rehabilitation. However, in addition to the possibility of relying on user-feedback to control stimulation intensity [89], measuring the electrical impedance of the skin may also serve as a method of control [90]. In this way, realtime feedback circuits can be incorporated into the design of devices thereby determining the intensity parameters of any applied stimulation based on properties of the skin.

\section{Spatial Resolution}

The smallest distance between two detectable points of contact on human skin (static touch) has also been used as a measure of tactile sensitivity, and has clear implications for the design of haptic devices, particularly those that rely on pin-arrays and shape memory alloy wires [82] [91]. Inter-point discrimination varies depending on which parts of the hand are being tested. Greatest sensitivity is reported on the volar surfaces of the thumb and first finger [92]. Although there is some variability between individuals, observations have been 
made that the smallest detectable distance between two points, applied simultaneously in this region, is approximately $1.88 \mathrm{~mm}$ [62] although this value increases with age [22]. In this way, simulation of a flat surface or object can be achieved by utilising contact points separated by distances which are below the two point discrimination threshold. Darghai et al [67] demonstrated that application of a $10 \times 15$ $1 \mathrm{~mm}$ actuator array may be used to convey a perception of a smooth object surface. It is worth noting that when two stimuli are presented successively, rather than simultaneously, the inter-point discrimination distance is considerably smaller which is attributed to the role of the Meisner corpuscles in active touch [67]. As a result, the design of haptic devices is dependent on the nature of the task being simulated.

\section{Future Directions}

Haptic rehabilitation is a rapidly growing field with the potential to offer exciting advances in rehabilitation of sensory and motor function. It is likely to be particularly important in hand rehabilitation because of the complex and integral connection between somatosensory feedback and motor dexterity needed to perform complicated manual tasks. This is particularly exciting given the relatively low recovery rates currently seen in the upper limb [40] [41] and recent research indicating that sensory stimulation may make an important contribution to sensory and motor recovery [44], [45].

There are, however, several issues which need to be considered and addressed if haptic rehabilitation is to live up to this potential. Haptic devices and virtual reality are new, complex and rapidly changing fields. Neurorehabilitation is also undergoing rapid changes in knowledge and practice so it is vital that developments in haptic rehabilitation have strong theoretical underpinnings and that the design and evaluation of devices adopts sound scientific principles. To this effect there are several recommendations which could guide researchers interested in this field.
Firstly there is a need for a more theoretical approach to the design and evaluation of haptic devices. The Medical Research Council [93] considers the development of underlying theory to be an important early stage in the design and evaluation of complex interventions. Designers therefore need to ensure they have a thorough understanding of the psychophysical properties of the two sensory systems and to develop and communicate clear rationales as to which sensory system(s) their device is targeting and why. This paper provides a framework to enable this process to be made more explicit in future work.

Evaluations of the effects of haptic technologies on neurorehabilitation also need to be more theoretical. For instance, most trials, to date, have not included any control groups making it difficult to ascertain whether improvements are due to the haptic technology or other factors. Moreover, there is often a distinct lack of information about the participants' sensory capacities or lesion locations before or after the studies. Studies which explore the mechanisms by which haptic feedback does or does not aid sensory-motor recovery are urgently needed. Incorporating accurate sensory profiling, perhaps including sensory evoked potentials, and exploring changes to sensory and motor regions of the brain using neurophysiological techniques, before, during and after haptic interventions would aid the science of this field, further device design and guide future rehabilitation paradigms.

This leads to a recommendation for greater interdisciplinary work in both the design and evaluation of haptic rehabilitation devices. Many existing devices were primarily designed by engineers with relatively limited input from rehabilitation experts or end-users in the early design processes. True interdisciplinary working with engineers, therapists, and psychologists working together with end-users from the early design phases is likely to result in devices which are more effective and suitable for home use. The research by Merrett et al [49] successfully adopted this approach to design finger worn tactile haptic actuators. 
Interdisciplinary evaluations are also crucial for both clarifying rehabilitation effectiveness and for explicating underlying recovery mechanisms associated with haptic feedback.

From a more pragmatic perspective there is an urgent need to develop devices which are cheaper, smaller and easier to use. The main rationale currently given for haptic VR is its potential to reduce the need for and costs of one-to-one therapy and to promote neuroplasticity through intensive practice. This will only be possible if devices are cheap enough for health services or individual patients to purchase, small enough to be unobtrusive in a home setting and simple enough to use without therapist support. In particular, careful thought needs to be given to how users with weak and often contracted hands can apply the devices. Complex exoskeletons and even simple gloves may be impossible for some people to don and doff and may prevent others from using the device sufficiently to promote plasticity. Thimble devices which sit over the finger-tips may provide one solution [49].

Tactile haptic technologies are not as well developed as kinaesthetic systems and research to develop tactile systems is urgently needed. This is especially relevant in hand rehabilitation both because the hand is a major tactile organ for exploring the environment and because of the need for tactile feedback in the performance of many skilled and dextrous movements. To date most tactile haptic devices have focused on vibration feedback, yet vibration is not a common tactile experience in daily activities. The development of tactile devices which can stimulate other modalities, especially pressure and temperature is recommended. Ultimately devices which are multi-modal will facilitate the realism and immersive nature of VR allowing people to experience, for instance, holding a warm cup of tea or using a cold metal drill.

Given the stated aim of producing realistic sensory perceptions of virtual objects there is also a need to develop robust methodologies for the evaluation of haptic experiences. Sensory perception is a subjective experience and, therefore, very difficult to quantify. However, if designers aim to mimic holding/touching everyday objects, methods for ascertaining the realism of those sensory perceptions are needed. To date few researchers have explored what sensory perceptions are generated by haptic devices focussing instead on the impact of haptic feedback on motor function [7]. Qualitative methods, such as unstructured interviews, semantic lexicons and photo elicitation can be useful in the early stages of device design to discover people's opinions and perceptions of the sensory stimuli [49]. These methods do not, however, allow for easy comparison between devices or for quantifying the degree to which sensory function changes. Methods used in textile science [94], [95] could be adapted to provide robust tools for these purposes.

\section{Conclusion}

Haptic rehabilitation offers the potential to advance sensorimotor hand rehabilitation but both scientific and pragmatic developments are needed to ensure that its potential is realised. This review provides a useful framework for rehabilitation therapists and engineers and highlights issues of importance in the design and evaluation of haptic devices.

\section{References}

[1] Alamri A, Eid M, Iglesias R, Shirmohammadi S, El Saddik A. Haptic Virtual Rehabilitation Exercises for Post-stroke Diagnosis. IEEE Transactions on Instrumentation \& Measurement. 2007; 57: 1876-1884.

[2] Ang Q-Z, Horan B, Najdovski Z, Nahavandi S. Grasping virtual objects with multi-point haptics. In IEEE Virtual Reality Conference; 2011 March 19-23; Singapore: 2011. 189-190 p.

[3] Harwin WS, Patton JL, Edgerton VR. Challenges and Opportunities for Robot-Mediated Neurorehabilitation. Proc of the IEEE. 2006; 94: 1717-1726.

[4] Markow T, Ramakrishnan N, Huang K, Starner T, Eicholtz M, Garrett S, Profita H, Scarlata A, Schooler C, Tarun A, Backus D. Mobile Music Touch: Vibration stimulus in hand rehabilitation. 4th International Conference on Pervasive Computing Technologies for Healthcare; 2010 March 22-25; Georgia, Atlanta. 1 - 8p 
[5] Nudo RJ, Plautz EJ, Milliken GW. Adaptive Plasticity in Primate Motor Cortex as a consequence of behavioural Experience and Neuronal Injury. Seminars in Neuroscience 1997; 9: 13-23.

[6] Jack D, Boian R, Merians AS, Tremaine M, Burdea GC, Adamovich SV, Recce M, Poizner H. Virtual realityenhanced stroke rehabilitation. IEEE Transactions on Neural Systems \& Rehabilitation Engineering. 2001; 9: 308-318.

[7] Adamovich SV, Merians A, Boian R, Tremaine M, Burdea G, Recce M, Poizner H. A virtual reality based exercise sytem for hand rehabilitation post-stroke: transfer to function. IEEE Engineering Medicine Biology Sociology. 2004; 7: 4936-9.

[8] Jones LA, Lederman SJ. Human Hand Function. New York: Oxford University Press: 2006.

[9] Connell LA. Sensory Impairment and Recovery after Stroke. PhD thesis, University of Nottingham, UK. 2006.

[10] Willis DW, Coggeshall RE. Sensory Mechanisms of the Spinal Cord: Volume 1: Primary Afferent Neurons and the Spinal Dorsal Horn: Primary Afferent Neurons and the Spinal Dorsal Horn, New York, Plenum Press; 1991.

[11] Gandevia SC, McClosky DI, Burke D. Kinaesthetic signals and muscle contraction. Trends in NeuroScience. 1992; 15: 62-65.

[12] Collins DF, Refshauge KM, Todd G, Gandevia SC. Cutaneous Receptors Contribute to Kinesthesia at the Index Finger, Elbow and Knee. Journal of Neurophysiology. 2005; 94: 1699-1706.

[13] Goble DJ, Coxon JP, Wenderoth N, Van Impe A, Swinnen SP. Proprioceptive sensibility in the elderly: Degeneration, functional consequences and plasticadaptive processes. Neuroscience \& Biobehavioral Reviews. 2009; 33: 271-278.

[14] Knoop MPM, Steultjens M, van der Leeden M, van der Esch CA, Thorstensson LD, Roord, W.F. Lems, J. Dekker, Proprioception in knee osteoarthritis: a narrative review. Osteoarthritis \& Cartilage. 2011; 19: 381-388.

[15] Shidahara H, Deie M, Niimoto T, Shimada N, Toriyama M, Adachi N, Hirata K, Urabe Y, Ochi M. Prospective Study of Kinesthesia after ACL Reconstruction. International Journal Sports Medicine 2011; 32: 386-392.

[16] Muaidi QI, Nicholson LL, Refshauge KM. Do elite athletes exhibit enhanced proprioceptive acuity, range and strength of knee rotation compared with non- athletes? Scandinavian Journal of Medicine \& Science in Sports 2009; 19: 103-112.

[17] Batson G. Update on Proprioception: Considerations for Dance Education. Journal of Dance Medicine \& Science. 2009; 13: 35-41.

[18] Rosenkranz K, Butler K, Williamon A, Rothwell JC. Regaining motor control in musician's dystonia by restoring sensorimotor organization. Journal of Neuroscience 2009; 29: 14627-14636.

[19] Halata Z, Baumann KI. Anatomy of Receptors. In: Grunwald M, editors. Human Haptic Perceptions: Basics and Applications. Birkhauser Verlag Basel. 2008.

[20] Puertas A, Pures P, Echenique AM, Graffigna JP, Ensinck G. Braille line using electrical stimulation. Journal of Physics: Conference series. 2007; 90: 1-7.

[21] Van Erp JBF, Van den Dobbelsteen JJ. On the design of tactile displays (Report TM-98-B012). Soesterberg, The Netherlands: TNO Human Factors Research Institute. 1998.

[22] Stevens JC, Choo KK. Spatial acuity of the body surface over the lifespan. Somatosensory and Motor Research. 1996; 13: 153-166.

[23] Darian-Smith I, Johnson KO. Thermal sensibility and thermoreceptors. Journal of Investigative Dermatology. 1977; 69: 146-153.

[24] Johansson RS. Sensory Guidance and Movement. Chichester: Wiley: 1998; 45-63p.

[25] Goebl W, Palmer C. Tactile feedback and timing accuracy in piano performance. Experimental Brain Research. 2008; 186: 471-479.

[26] Palmer C, Koopmans E, Loehr JD and Carter C. Movement-Related Feedback and Temporal Accuracy in Clarinet Performance. Music Perception: An Interdisciplinary Journal. 2009; 26: 439-449.

[27] Tyson, S, Hanley, M, Chillala, J, Selley, AB and Tallis, RC. Sensory loss in hospital-admitted people with stroke: characteristics, associated factors, and relationship with function. Neurorehabilitation and Neural Repair. 2008; 22: 166-172.

[28] Yekutiel M. Sensory Re-education of the Hand after Stroke. London: Whurr; 2000.

[29] Carey L. Somatosensory loss after stroke. Critical Reviews in Physical \& Rehabilitation Medicine. 1995; 7 : 51-91.

[30] Ghez C, Gordon J, Ghilardi MF, Christakos CN, Cooper SE. Roles of proprioceptive input in the 
programming of arm trajectories. Cold Spring Harb Symp Quant Biol. 1990; 55: 837-847.

[31] Roland PE, Larsen B, Lassen NA, Skinhof E. Supplementary motor area and other cortical areas in organization of voluntary movements in man. Journal of Neurophysiology. 1980; 43: 118-36.

[32] Collins DF, Cameron T, Gillard DM, Prochazka A. Muscular sense is attenuated when humans move. Journal of Physiology. 1998; 508: 635-643.

[33] Bastian AJ. Mechanisms of Ataxia. Physical Therapy. 1997; 77: 672-675.

[34] Smania N, Montagnana B, Faccioli S, Fiaschi A, Aglioti SM. Rehabilitation of Somatic Sensation and related deficits of motor control in patients with pure sensory stroke. Archives of Physical Medicine and Rehabilitation 2003;84:1692-702.

[35] Kim JS, Bae YH. Pure or predominant sensory stroke due to brain stem lesion. Stroke. 1997; 28: 17611764 .

[36] Sainburg RL, Poizner H, Ghez C. Loss of Proprioception produces Deficits in Interjoint Coordination. Journal of Neurophysiology. 1993; 70: 2136-47.

[37] Westling G, Johansson RS. Factors influencing the force control during precision grip. Experimental Brain Research 1984; 53:277-284.

[38] Agioloti S, Beltramello A, Bonazzi A, Corbetta M. Thumb-pointing in humans after damage to somatic cortex. Experiemental Brain Research, 1996. 109: 92-100.

[39] Kwakkel G, Wagenaar R, Kollen B, Lankhorst G. Predicting disability in Stroke - a critical review of the literature. Age and Ageing 1996; 25: 479-489.

[40] Wade DT, Langton-Hewer R, Wood VA, Skilbeck CE, Ismail HM. The hemiplegic arm after stroke: measurement and recovery. J. of Neurology, Neurosurg. \& Psych. 1983; 46: 521-524.

[41] Sunderland A, Tinson DJ, Bradley EL, Fletcher D, Langton-Hewer R, Wade DT. Enhanced physical therapy improves recovery of arm function after stroke. A randomised control trial. J. of Neurology, Neurosurg. \& Psych. 1992; 55: 530-535.

[42] Byl N, Roderick J, Mohamed O, Hanny M, Kotler J, Smith A, Tang M, Abrams G. Effectiveness of Sensory and Motor Rehabilitation of the Upper Limb Following the Principles of Neuroplasticity: Patients Stable Poststroke. Neurorehabilitation \& Neural Repair. 2003; 17: 176-191.
[43] Doyle S, Bennett S, Fasoli SE, McKenna KT. Interventions for sensory impairment in the upper limb after stroke. Cochrane Database of Systematic Reviews 2010, Issue 6. Art. No.: CD006331. DOI: 10.1002/ 14651858.CD006 331.pub2

[44] Richards LG, Stewart KC, Woodbury ML, Senesac C, Cauraugh JH. Movement-dependent stroke recovery: A systematic review and meta-analysis of TMS and fMRI evidence. Neuropsychologia. 2008; 46: 3-11.

[45] Smith PS, Dinse HR, Kalisch T, Johnson M, Walker-Batson D. Effects of Repetitive Electrical Stimulation to Treat Sensory Loss in Persons Poststroke. Archives of Physical Medicine Rehabilitation. 2009; 90: 2108-2111.

[46] Cuypers K, Levin O, Thijs H, Swinnen SP, Meesen RLJ. Long-term TENS treatment improves tactile sensitivity in MS patients. Neural Rehab. \& Neural Repair. 2010; 24: 420-427.

[47] Cao M-L, Zhang J-Z Effect of early rehabilitation therapy on the rehabilitation of limb sensation and muscle strength in patients with incomplete spinal cord injury Zhongguo Linchuang Kangfu, , 2004; 8: 5473-5.

[48] Sallnäs E, Rassmus-Gröhn K, Sjöström C. Supporting presence in collaborative environments by haptic force feedback. ACM Transactions on ComputerHuman Interaction. 2000; 7: 461- 476.

[49] Merrett GV, Metcalf CD, Zheng D, Cunningham S, Barrow $S$ and Demain SH. Design and Qualitative Evaluation of Tactile Devices for Stroke Rehabilitation. IET Assisted Living Conference; 2011 April 6; London. $1-6 \mathrm{p}$.

[50] McLaughlin M, Rizzo A, Jung Y, Peng W, Yeh SG, Zhu W. Haptics- Enhanced Virtual Environments for Stroke Rehabilitation. IPSI Conference; 2005 Jun 2-5. Cambridge.

[51] Takahashi CD, Der-Yeghiaian L, Le VH, Cramer SC. A robotic device for hand motor therapy after stroke. IEEE International Conference on Rehabilitation and Robotics; 2005 Jun 28 - Jul 1, Chicago. 17-20p.

[52] Frisoli A, Rocchi F, Marcheschi S, Dettori A, Salsedo F, Bergamasco M. [Internet]. A new forcefeedback arm exoskeleton for haptic interaction in virtual environments. Washington, DC: IEEE Computer Society. [Cited 2010 November 24]; Available from: http://dl.acm.org/citation.cfm?id=1050037

[53] CyberGloveSystems [Internet]. San Jose, CA: CyberGlove Systems LLC; [cited 2010 November 24]; Available from: http://www.cyberglovesystems.com/sites/default/files/Cy berTouch_Brochure_2009.pdf 
[54] Palazzolo J, Ferraro M, Krebs H, Lynch D, Volpe B, Hogan N. Stochastic estimation of arm mechanical impedence during robotic stroke rehabilitation. IEEE Transactions on Neural Systems and Rehabilitation Engineering. 2007; 15: 94-103.

[55] Dovat L, Lambercy O, Ruffieux Y, Chapuis D, Gassert R, Bleuler H, Teo C, Burdet E. A haptic knob for rehabilitation of stroke patients. IEEE International Confernce on Intelligent Robots and Systems; 2006 Oct; Beijing. 977-982 p.

[56] Sensable [Internet]. Wilmington, MA: Sensable; [cited 2011 September 30]; Available from: http://www.sensable.com/products-haptic-devices.htm

[57] Salisbury K, Brock D, Massie T, Swaruo N, Zilles C. Haptic rendering: Programming touch interaction with virtual objects. Symposium on Interactive 3D Graphics; 1995 Apr 9-12; Monterey. 123-130 p.

[58] Miyahra K, Okada Y. A surgical simulation system supporting COLLADA-based file format. Fifth International Conference on Computer Graphics, Imaging and Visualisation; 2008 26-28 Aug; Penang. 126-131 p.

[59] Merians AS, Poizner H, Boian R, Burdea GC, Adamovich SV, Sensorimotor training in a virtual reality environment: does it improve functional recovery poststroke? Neurorehabilitation and Neural Repair. 2006; 20: 252-267.

[60] Qui Q, Fluet GG, Saleh S, Lafond I, Merians AS, Adamovich SV. Integrated Versus Isolated Training of the Hemiparetic Upper Extremity in Haptically Rendered Virtual Environments. Conf Proc IEEE Eng Med Biol Soc. 2010 Aug 31 -Sept 4; Buenos Aires. 20102255 2258 p.

[61] Vo DM, Vance, JM, Marasinghe M. Assessment of haptics-based interaction for assembly tasks in virtual reality. World Haptics Conference Proceedings; 2009 Mar 18-20; Salt Lake City. 494-499 p.

[62] Qi W, Taylor R, Healey C, Martens JB. A comparison of immersive HMD, fish tank VR and fish tank with haptic displays for volume visualization. The 3rd Symposium on Applied Perception in Graphics and Visualization, 2006 Jul 28-30; Boston; 2006, 51-58 p.

[63] Chouvardas VG, Miliou AN, Hatalis MK. Tactile Displays: Overview and recent advances. Displays. 2008; 29: 185-194.

[64] Sherrick CE, Cholewiak RW, Collins AA. The localization of low and high frequency vibrotactile stimuli. Journal of the Acoustical Society of America 1990; 88: 169-179.

[65] Ferrari De Castro MC, Cliquet A.
Artificial Sensorimotor Integration in Spinal Cord Injured Subjects Through Neuromuscular and Electrotactile Stimulation. Artificial Organs 2000; 24: 710-7.

[66] Jiang L, Cutkosky MR, Ruutiainen J, Raisamo R. Using Haptic Feedback to Improve Grasp Force Control in Multiple Sclerosis Patients. IEEE transactions on Robotics 2009; 25: 593 - 60 .

[67] Dargahi J, Najarian S. Human tactile perception as a standard for artificial tactile sensing - a review. International Journal of Medical Robotics and Computer Assisted Surgery. 2004; 1: 23-35.

[68] Pasterkamp E. Mechanoreceptors in the Glabrous Skin of the Human Hand. Archives of Physiology and Biochemistry 1999; 107: 338-341.

[69] Fisch A, Mavroidis C, Melli-Huber J, Bar-Cohen Y. Haptic devices for virtual reality, telepresence and human-assistive robotics. In: Biologically-inspired intelligent robots. Bar-Cohen Y, Breazeal CL. Editors. Bellingham; WA: SPIE Press; 2003; Chapter 4.

[70] Verrillo RT. Psychophysics of vibrotactile stimulation. Journal of Acoustical Society of America 1985; 77: 225-232.

[71] Van Erp JBF, Van den Dobbelsteen JJ. On the design of tactile displays (Report TM-98-B012). Soesterberg, The Netherlands: TNO Human Factors Research Institute. 1998.

[72] Cleary AG, McKendrick H, Sills JA. Hand-arm vibration syndrome may be associated with prolonged use of vibration computer games. British Medical Journal. 2002; 324: 301.

[73] Takeuchi T, Futatsuka M, Imanish H, Yamada S. Pathological changes observed in the finger biopsy of patients with vibration-induced white finger. Scandinavian Journal of Work, Environment and Health. 1986; 12: 280-283.

[74] Chang D. Haptics: gaming's new sensation. IEEE Computer. 2002; 35: 84-86.

[75] Lee CJ. Hacking the Nintendo Wii Remote. IEEE Pervasive Computing. 2008; 7: 39-45.

[76] Hashimoto $\mathrm{Y}$, Watanabe J, Maeda T, Ando $\mathrm{H}$. Tactile Illusion of Texture using Vibration to a Finger for Active Touch. IEEE World Haptics. 2011 June 21-24; Istanbul, Turkey.

[77] Hoshi T, Iwamoto T, Shinoda, H. Non-contact Tactile Sensation Synthesized by Ultrasound Transducers. Eurohaptics Conference. 2009 March 18-20: Salt Lake City, UT; 2009. 256-260 p. 
[78] Birke JA, Sims DS. Plantar sensory threshold in the ulcerative foot. Leprosy Review 1986; 57: 261-267.

[79] Minamizawa K, Fukamachi S, Kawakami N, Tachi $\mathrm{S}$. Interactive representation of virtual object in hand-held box by finger-worn haptic display . Haptic interfaces for virtual environment and teleoperator systems. 2008; Mar 13-14: 367-368.

[80] Taylor PM, Pollet DM, Hosseini-Sianaki A, Varley CJ. Advances in an electrorheological fluid based tactile array. Displays. 1998; 18: 135-141.

[81] Iwamoto T, Tatezono M, Shinoda H. Haptics: Perception, Devices and Scenarios.

Lecture Notes in Computer Science. 2008; 5024: 504513.

[82] Taylor PM, Moser A, Creed A. A sixty-four element tactile display using shape memory alloy wires. Displays. 1998; 18: 163-168.

[83] Scheibe R, Moehring M, Froehlich B. Tactile Feedback at the Finger Tips for Improved Direct Interaction in Immersive Environments. IEEE Symposium on 3D User Interfaces; 2007 Mar 10-11; Charlotte. 125-132 p.

[84] Ho HN, Jones LA. Contribution of thermal cues to material discrimination and localization. Perception \& Psychophysics. 2006; 68: 118-128.

[85] Szeto AYJ, Saunders FA. Electrocutaneous Stimulation for Sensory Communication in Rehabilitation Engineering. IEEE Transactions on Biomedical Engineering. 1982; 4: 300-308.

[86] Davey NJ, Nowicky AV, Zaman R. Somatotopy of perceptual threshold to cutaneous electrical stimulation in man. Experimental Physiology. 2001; 86: 127-130.

[87] Peurala, SH, Pitkänen K, Sivenius J, Tarkka IM. Cutaneous electrical stimulation may enhance recovery in chronic stroke. Clinical Rehabilitation. 2002; 16: 709716.

[88] Geldard FA. The Human Senses. $2^{\text {nd }}$ ed. New York: John Wiley \& Sons Inc.; 1972.

[89] Kajimoto H, Kawakami N, Maeda T, Tachi S. Electro-tactile display with force feedback. In World Multi conference on Systemics, Cybernetics and Informatics. Orlando, US; 2001.

[90] Kajimoto H. Electro-tactile Display with Real-Time Impedance Feedback. In Kappers et al. (Eds.): EuroHaptics (2010), part 1, 285-291. Berlin: Springer Verlag; 2010.

[91] Matsunaga T, Totsu K, Esashi M, Haga Y. Tactile display for 2-D and 3-D shape expression using SMA micro actuators. $3^{\text {rd }}$ International IEEE Special Topic Conference on Microtechnologies in Medicine and Biology; 2005 May 12-15; Honolulu, 88-91 p.

[92] Nolan MF. Two-point discrimination assessment in the upper limb in young adult men and women. Physical Therapy. 1982; 62: 965-969.

[93] Medical Research Council [Internet]. Developing and evaluating complex interventions: new guidance. London: Medical Research Council; [cited 2011 September 30]; Available from: www.mrc.ac.uk/complexinterventionsguidance.pdf

[94] Childs THC, Henson B. Human tactile perception of screen-printed surfaces: self-report and contact mechanics experiments. J. Eng. Tribology. 2007; 221: 427-441.

[95] Chen X, Shao F, Barnes C, Childs T, Henson B. Exploring relationships between touch perception and surface physical properties. Int. J. of Design. 2009; 3: 6776. 